\title{
An Exploratory Study on the Sustainability of Emerging Small-to-Medium Enterprises Ecosystems
}

\author{
Pillar Benedetti Vasconcellos Luz ${ }^{1}$, Juliana Fernandes ${ }^{1,2}$, George Valença ${ }^{3}$, \\ Rodrigo Pereira dos Santos ${ }^{1}$
}

\author{
${ }^{1}$ Department of Applied Informatics \\ Federal University of the State of Rio de Janeiro (UNIRIO) \\ Rio de Janeiro, RJ - Brazil \\ ${ }^{2}$ Federal Institute of Piauí (IFPI) \\ Campo Maior, PI - Brazil \\ ${ }^{3}$ Computing Department \\ Federal Rural University of Pernambuco (UFRPE) \\ Recife, PE - Brazil \\ \{pillar.luz, juliana.costa, rps\}@uniriotec.br, george.valenca@ufrpe.br
}

\begin{abstract}
A recent trend of small-to-medium enterprises (SME) is their close collaboration to create new products and reach new market niches. In this context, sustainability emerges as an important requirement of an ecosystem formed by companies (actors), mainly thinking of its longevity and survival. In this paper, we modeled and analyzed a real case of an emerging SME software ecosystem (SECO) based on a system sustainability framework in order to model and analyze sustainability as a SECO requirement. We also applied a set of SECO factors to the produced model, considering existing relationships between actors. Results demonstrate the importance of analyzing this requirement to assist an emerging SECO from the beginning, but also show the difficulty in treating sustainability as a requirement.
\end{abstract}

Keywords. Software Ecosystems; Sustainability; SME.

\section{Introduction}

A software ecosystem (SECO) is often defined as a group of actors working as a unit and interacting with a shared market for software and services, centered on a common technological platform (Jansen et al. 2009). It supports social web since plays a central role enabling interactions through a platform. The effort and costs required to maintain complex software systems in SECO are often high, involving continuous refactoring to ensure longevity in the face of changing requirements. According Capilla et al. (2017), the longevity of a system affects its sustainability, which can be understood as meeting current needs without compromising the ability of future generations to meet their own needs. However, there is a lack of understanding on how to treat sustainability as a requirement of software systems, especially when it comes to a SECO.

In this scenario, it is challenging to treat sustainability as a SECO requirement in order to analyze the ecosystem health, especially because there are few research initiatives addressing such subject. In addition, there is a claim for software engineering and training on sustainability as a requirement of information systems (Lago and 
Penzenstadler 2017), e.g. in the context of small-to-medium enterprises (SME) in which companies that collaborate to create new products and reach new market niches.

In this paper, we investigate an emerging SECO formed by SMEs. We recognize the increasing alliances among SMEs as growth strategies. Hence, we decided to model a real ecosystem case based on a system sustainability framework. We also verified the relevance of sustainability in this context based on verifying a set of 15 technical, human, and organizational factors compiled by Santos et al. (2016). The resulting models were analyzed by an expert in SME SECO and later evaluated via a semistructured interview. Although it was possible to notice the relevance of analyzing sustainability in a SECO, it was not easy to do so. However, such difficulty was expected due to the lack of research addressing this topic (Barbosa et al. 2013).

This paper is organized as follows: Section 2 presents the background; in Section 3, we describe the system sustainability framework from Lago el al. (2015) and its application in a real case of an emerging SME SECO; Section 4 presents an analysis on how SECO factors from Santos et al. (2016) were observed in the real case; Section 5 discusses the evaluation and interview conducted with an SME SECO expert; finally, in Section 6, we conclude the paper with the main contributions and limitations of this research, as well as pointing out future work.

\section{Background}

\subsection{Software Ecosystems}

Bosch and Bosch-Sijtsema (2010) state that a SECO consists of a set of software solutions that aid and automate activities and transactions of actors associated with a social or business ecosystem. In Figure 1, we present the most common elements that form a SECO together with their relationships. In a SECO, a platform is often provided to support the creation of products and services. These solutions can be included, modified or extended as software artifacts. An ecosystem also has a community of hubs (e.g. leading agents) and niche players (i.e. all stakeholders who affect the ecosystem through individual actions, such as contributing to the platform). Both participants play specific roles in this community, e.g. end-user or developer.

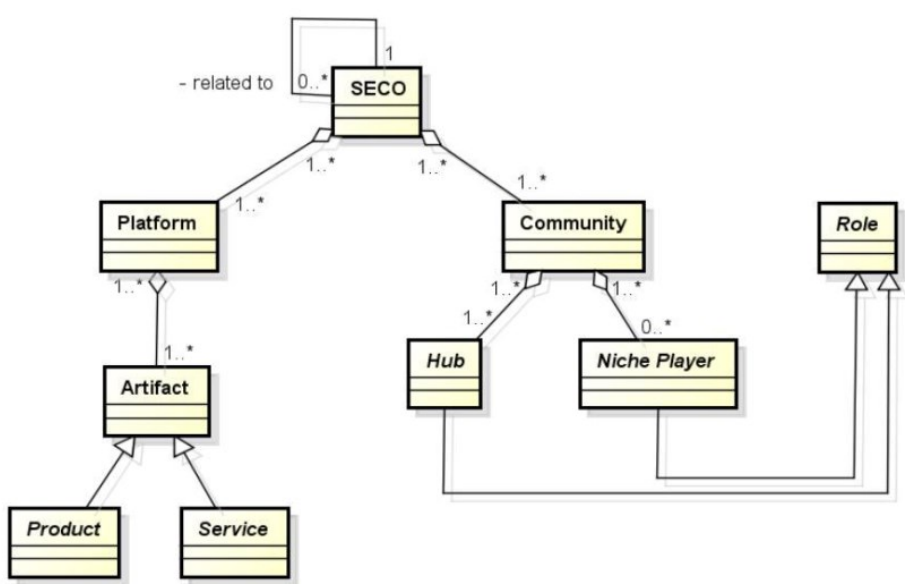

Figure 1. SECO actors and their relationships. Source: (Santos 2016)

The relationships between a common technological platform and its actors can be analyzed from a set of technical, human and organizational factors affecting an 
ecosystem (Santos et al. 2016), as shown in Table 1. According to Santos et al. (2016), the first factor (F1) deals with the management of diversity of organizations and relationships in a SECO, whose concern is the identification and understanding of the roles that each actor must play within SECO. The next factor (F2) brings to the light possible contribution of external developers acting on a central platform and what actions must be taken into consideration when maintaining SECO performance. In turn, the third factor (F3) focuses mainly on the sharing of content, knowledge, problems, experiences, and skills. The promotion of reusability is decisive in the fourth factor (F4), which deals with improvements in software reuse in the scenario of global software engineering, since a SECO appears as a promising approach to this.

Table 1. Technical, human and organizational factors affecting a SECO.

Source: (Santos et al. 2016)

\begin{tabular}{cl}
\hline Factor & \multicolumn{1}{c}{ Description } \\
\hline F1 & Deal with diversity of organizations and relationships within a SECO \\
\hline F2 & Encourage external developers to use a common technological platform \\
\hline F3 & Share content, knowledge, problems, experiences and abilities \\
\hline F4 & Improve software reuse in the scenario of global software engineering \\
\hline F5 & Reposition organizations to act as network actors and reduce their internal workforce \\
\hline F6 & Consider diversity of new functionalities offered to clients \\
\hline F7 & Invest in transparency \\
\hline F8 & Support modular system design \\
\hline F9 & Support organization openness \\
\hline F10 & Define internal characteristics related to SECO health and stability \\
\hline F11 & Define well-established SECO scope/boundaries \\
\hline F12 & Identify capacities and relationships between actors within a SECO \\
\hline F13 & Have a clear definition of the process \\
\hline F14 & Strengthen a communicative character inherent for programming activities \\
\hline F15 & Ensure compliance based on the characteristics of different application domains
\end{tabular}

According to Bosch and Bosch-Sijtsema (2010), organizations want to reposition themselves to act as network actors and reduce their internal workforce, as the fifth factor (F5) highlights. An example of this effort involves the sixth factor (F6), which focuses on new features available to customers, i.e. what can be observed in app stores. To ensure that there is transparency, a SECO must provide information related to the platform development, as highlighted by the seventh factor (F7). This factor is aligned with the eighth factor - modular system design (F8) - that applies engineering principle: decompose a system into manageable parts to develop from modules.

When dealing with the organization opening in the ninth factor (F9), we refer to a critical factor for knowledge sharing. The definition of internal characteristics related to SECO health and stability is pointed out in the tenth factor (F10) and targets SECO participants and everything that involves them, e.g. size, roles, connectivity etc. SECO should have well defined boundaries (market, technology, infrastructure, or company), as referred in the eleventh factor (F11). The twelfth factor (F12) highlights the importance of taking care of any inconsistencies caused by evolutions and therefore reinforces the identification of capacities and relationships among SECO actors.

SECO evolutions demand a clear definition of processes as stated by the thirteenth factor (F13). Since new external actors are involved in the development process and need to work with 'traditional' actors, strengthening the communicative character inherent to programming has a great level of importance, as represented in the fourteenth factor (F14). Finally, fifteenth factor (F15) claims that management should 
be done in a centralized way, but recognizing that the community keeps the SECO providing demands and solutions for the platform (Fernandes and Santos 2017).

\subsection{Sustainability}

Hilty et al. (2006) define sustainability as the ability to support and preserve the function of a system over a period of time. In other words, the longevity of a platform is directly related to sustainability. According to Lago et al. (2015), sustainability can only be achieved when four dimensions are considered: economic, social, environmental, and technical. In Figure 2, we present the system sustainability framework of Lago et al. (2015), whose structure is extended from ISO/IEC 42030 - Architecture Assessment. This framework specifies relevant elements that characterize sustainability in a software system, assisting in the visual identification of its dimensions, as well as easing the understanding of the influence among its elements.

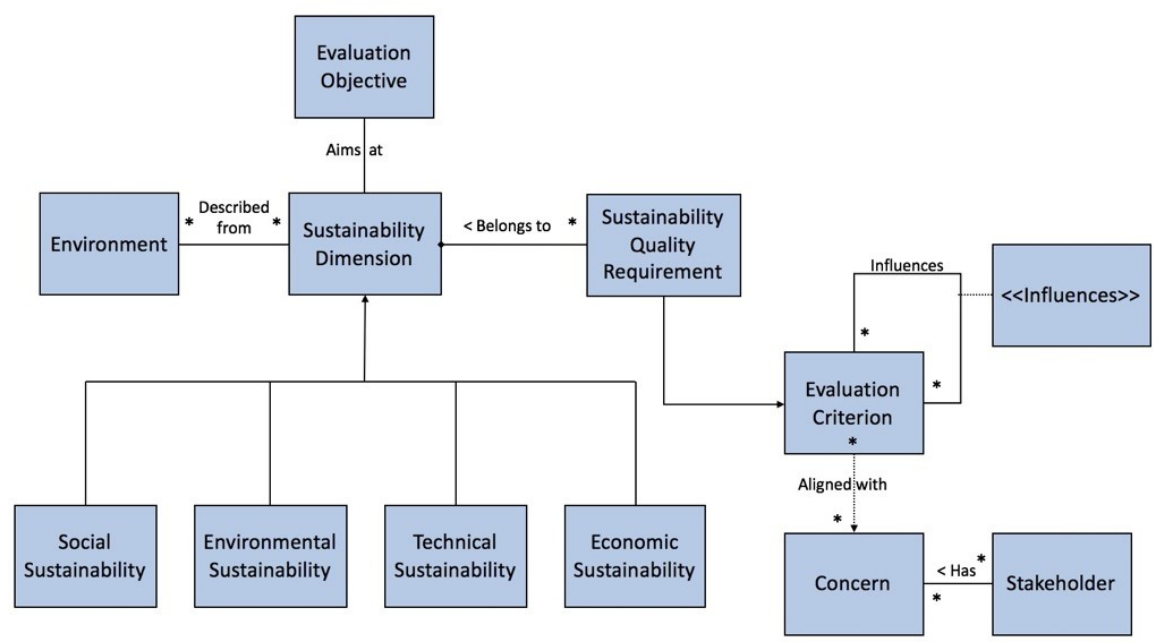

Figure 2. Structure of the system sustainability framework of Lago et al. (2015).

The goal of the framework is to identify to what extent the sustainability components influence each other. To evaluate sustainability of an ecosystem, we must consider a set of quality requirements, as well as criteria for evaluation. Since these criteria can influence the system requirements and, as a consequence, all sustainability dimensions, they should be aligned with the concerns of all stakeholders. With that said, it is clear that the whole framework should be considered when collecting data for sustainability assessment.

Considering the sustainability dimensions, social sustainability aims to ensure that future generations access social resources. It supports communities in any domain, activity or process that directly or indirectly creates social benefits. Environmental sustainability is concerned with ecological requirements, e.g. energy efficiency and ecological awareness. Technical sustainability focuses on embracing frequent changes and updates that exist in the technological world, without harming its longevity. Finally, economic sustainability aims to define strategies to save capital and generate value.

\section{Framing Sustainability}

This section presents the application of the system sustainability framework proposed by Lago et al. (2015) in the context of a SECO case. We can apply this framework to a SECO software platform as we apply to software systems because software is the 
common element. The difference lies in the borders since a SECO is open and a software system might not be. The resulting sustainability model presents four different sustainability dimensions, represented by small boxes. It also shows their parameters and evaluation methods. Based on these requirements, parameters are related to each other through positive and negative relationships, denoted by $<<$ influences $>>$ (i.e. a positive influence means that parameters can leverage each other). Figure 3 provides an example of how it is represented in the model. Parameters and evaluations are not, in essence, positive or negative; they are metrics.

\begin{tabular}{|c|c|c|c|}
\hline $\begin{array}{c}\text { Social } \\
\text { Dimension }\end{array}$ & $\begin{array}{c}\text { Environmental } \\
\text { Dimension }\end{array}$ & $\begin{array}{l}\text { Technical } \\
\text { Dimension }\end{array}$ & $\begin{array}{l}\text { Economic } \\
\text { Dimension }\end{array}$ \\
\hline $\begin{array}{c}\text { Sustainability Quality } \\
\text { Requirements }\end{array}$ & $\begin{array}{l}\text { Sustainability Quality } \\
\text { Requirements }\end{array}$ & $\begin{array}{c}\text { Sustainability Quality } \\
\text { Requirements }\end{array}$ & $\begin{array}{c}\text { Sustainability Quality } \\
\text { Requirements }\end{array}$ \\
\hline + Parameter & + Parameter & + Parameter & + Parameter \\
\hline - Evaluation & - Evaluation & - Evaluation & - Evaluation \\
\hline
\end{tabular}

Figure 3. System sustainability framework elements.

In Figure 4, we instantiate the system sustainability framework to the case of an emerging SME SECO. By applying the framework, we could analyze specific requirements, their parameters and how they should be evaluated. When looking at the social dimension, we know that interaction between organizations affects the quality of products and services in order to ensure a smooth operation of projects together. Internal satisfaction of each actor participating in the ecosystem could be measured throughout the change period as well as the frequency defined by the organization, so as to act on the improvements. Changes often affect the environment (and not everyone who plays will be willing and open to them). On the environmental dimension, meetings between emerging SECO organizations should exist, impacting the environment with respect to pollution and consumption of diesel oil. In addition, as demand increases, electricity consumption will increase, as it will require more and/or longer working environment.

In technical terms, the efficiency and reliability of the delivered product will be measured according to the existing delivery time standards prior to SECO and product stability if it is an application developed by the service provider, for example. There should also be a technical adaptation, which could lead to expansion of services if the expertise level is high. According to these aspects and considering the environment presented by Valença et al. (2014), it will be possible to evaluate the services provided through a customer satisfaction survey research.

In this context, such survey research will be a direct result of the economic dimension because the profitability will depend on the effectiveness of production from the contracted projects. Another impact of this dimension refers to the employees' expenses with urban movements, either for project meetings with the contracting companies or with other partner organizations of SECO. With the expansion (and possible dominance) of the market, as a result of an increase in demand, the quantity of services will increase, bringing more revenue for the involved organizations. 


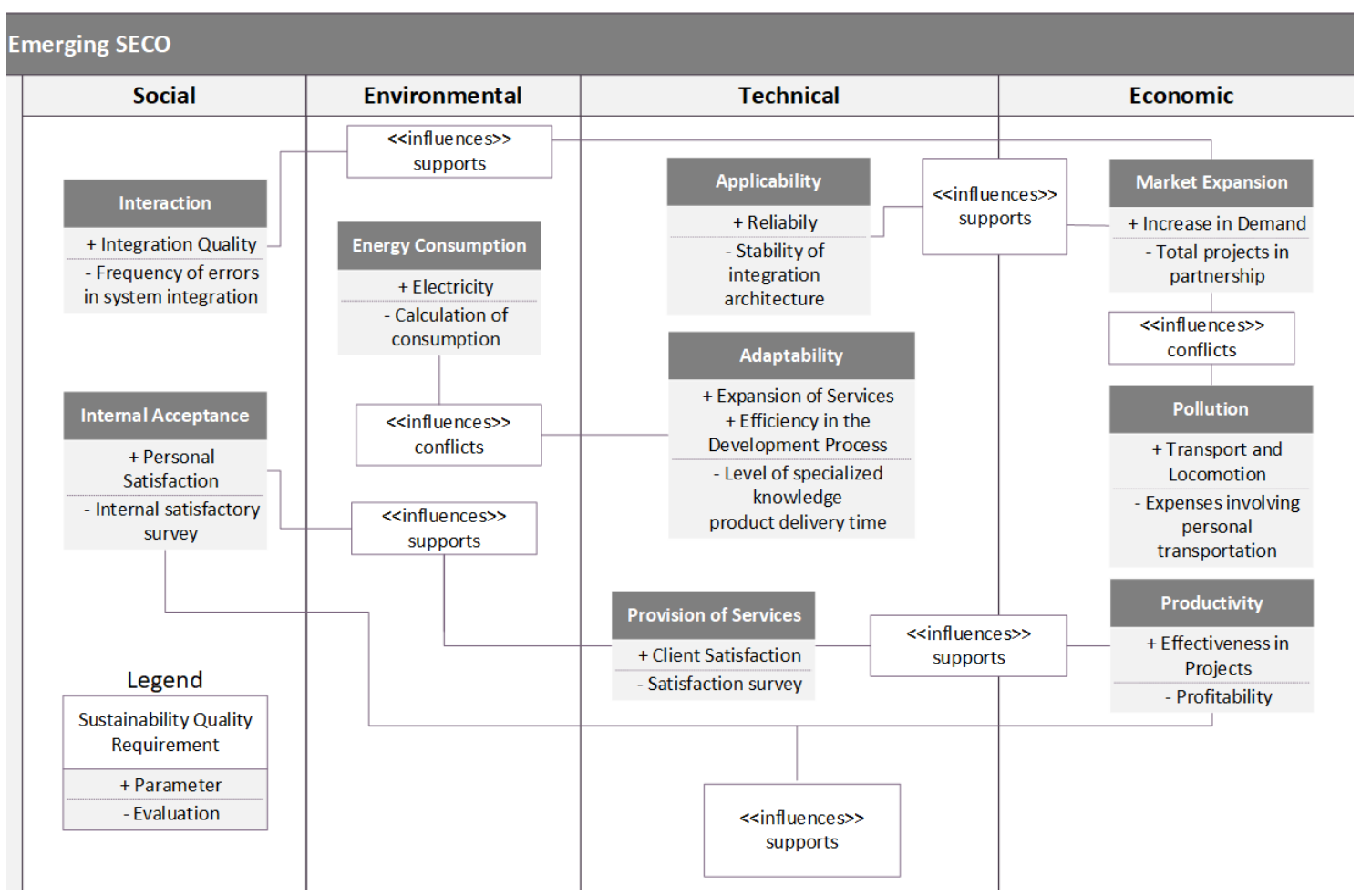

Figure 4. System sustainability framework applied to the emerging SME SECO.

\section{Analysis of SECO Factors}

Among the factors that could possibly affect relationships in an emerging SME SECO, we observed the diversity of organizations and relationships (F1), considering the organizational difference between the two companies that are working together on such class of SECO. In addition, different functionalities are presented to clients (F6): one of the goals for such SECO to become real is the possibility to present new functionalities to clients. We can also observe attention to programming aspects (F14), since developers from different companies must be aligned to deliver new solutions that communicate with each other.

Several SECO factors were partially observed. It is the case of sharing content, knowledge, problems, experiences, and skills (F3), which already appear in an emerging SME SECO, but are not yet fully seen (i.e. experiences will not necessarily be shared among organizations, and it may not exist before the emergence of a SECO). In addition, there is an idea of improving software reuse in the global software engineering (F4) scenario and repositioning organizations that focus on reducing the internal workforce as well, acting as network actors (F5).

Still regarding what has been partially observed, it is known that there is a need for transparency (F7), but with not much guidance to know how far to act in this way, since the organization opening (F9) is not an intangible factor and can be exploited. SECO scopes and frontiers are still unclear (F11), as well as the identification of capacity and relationships among actors in the SECO in question (F12), which make it difficult to define processes clearly (F13). Finally, the application domains with respect to their characteristics cannot be completely observed yet (F15). 
The SECO factors that could not be observed during this research include the encouragement of external developers to use the platform (F2), modular system design (F8) and definition of internal characteristics that are related to SECO health and stability (F10). These factors could not be observed because SECO is still forming itself, possibly with no attention being paid to more detailed points within the environment (i.e. defining characteristics involving health and stability), or requiring a stable, open platform for external developers.

\section{Evaluation}

In order to evaluate our findings (cf. Sections 3 and 4), we conducted an interview with an expert on an emerging SME SECO. The following three questions were asked (Table 2) to verify whether our results were in accordance with the existing reality of such type of ecosystem.

Table 2. Interview questions.

\begin{tabular}{c|l}
\hline Q1 & $\begin{array}{l}\text { Is the analysis presented consistent with the SECO case? Are the mentioned points aligned } \\
\text { with the reality of such SECO? If not, what would be the proper form of explanation? }\end{array}$ \\
\hline $\mathbf{Q 2}$ & $\begin{array}{l}\text { Does the system sustainability framework applied to SECO elements (parameters and } \\
\text { evaluation methods) referring to social, environmental, technical and economic dimensions? } \\
\text { Do such elements have consistent evaluation methods? Are the relations of influence } \\
\text { between the elements pointed out correct? }\end{array}$ \\
\hline Q3 & Suggestions and recommendations.
\end{tabular}

We adopted Goal-Question-Metric (GQM) approach (Basili 1992). We seek to verify the analysis we have performed on an emerging SME SECO. Table 3 describes how we considered GQM approach.

Table 3. GQM method.

\begin{tabular}{r|l}
\hline analyze & system sustainability framework and SECO factors affecting longevity \\
\hline with the objective of & characterize \\
\hline with respect to & modeling and analysis elements of the studied SECO \\
\hline from the point of view of & researchers \\
\hline in the context of & real SECO cases \\
\hline
\end{tabular}

We selected the participant by convenience, considering his/her extensive knowledge regarding emerging SME SECO: this fact was confirmed in the light of several scientific papers published by the participant. The main goal of the evaluation was to understand whether the system sustainability framework in conjunction with SECO factors contribute to model and analyze sustainability as a SECO requirement. We used a consent form to state the purpose of the study as well as the participants' responsibilities and rights. In addition, we reinforced data confidentiality. The participant had access to selected parts of the real, emerging SME SECO case and to a questionnaire had to be answered after reading and understanding the material. Qualitative information on the execution of the study was collected, as well as improvement suggestions based on the evaluation experience.

The instruments used to perform the study were prepared in MS Word 2016. On December 21st, 2017, the participant received an e-consent form containing a brief 
explanation of the work and the confidentiality agreement. After acceptance and return of the signed document (December 27th, 2017), a document containing the selected SME SECO model and analysis based on the framework was sent out (again by e-mail).

At that moment, the answers were given by the expert according to his/her understanding from what has been presented. The questionnaire was available for seven days. The result was received by e-mail on January 9th, 2018, with comments, but the questionnaire itself was not answered. After asking the participant to review the model on February 9th, 2018, the questionnaire was received. This result is presented in detail in Section 5.3, after reading, understanding and analyzing the received data.

Most of the answers were regarding changes that could be helpful. Since it is an emerging SME SECO, there are some integrations that are not common when studying other SECO environment, considered the traditional ones. There was a question about having the same parameters at two different dimensions: "Why is "pollution" in two dimensions? Should not it be only environmental?" - it made sense, but not in that way. Considering that this emerging SME SECO does not require much physical contact, it is not correct to consider it when thinking about environment. On the other hand, thinking about a financial situation, any moves to reunite teams would need an effort from both parts and it would affect the economic side.

There was also a suggestion pointed in Q2 where the participant advised that the integrations between parameters are not clear and the use of plus $(+)$ and minus $(-)$ can create some confusion. In order to better understand the issues raised in the study, an interview was conducted. This interview was scheduled via e-mail and made effective via Skype on April 25th, 2018. Five questions were asked, all based on the result of the evaluation, as shown in Table 4. The responses were recorded during the interview.

Table 4. Questions used on the interview.

Q1 In general, do you believe that the system sustainability framework instantiation applies to the case of this SECO? If not, why?

Q2 Was it clear that two different methods were used to conduct the study? In this case, a framework for modeling/analysis and SECO factors. If not, why?

Q3 Is the "integration architecture stability" setting related to the evaluation inserted within the applicability (technical dimension) requirement?

Q4 What name do you think we should give to this SECO? (considering that "Emerging SECO" was not a good choice)

Q5 Clarification and Acknowledgments.

Regarding the first question, the participant explained that "some items are not feasible to explore (we will not necessarily have data)" and "it is not necessarily useful to evaluate some pointed issues for the environment being evaluated". This refers directly to the fact that there are blind spots on the environmental dimension: it is mostly speculation due to the frequent changes and adaptations this SECO has. According to the participant, "there was not much relationship between SECO factors and ecosystem modeling". This is correct: both methods are different and have different purposes but can be complementary for analyzing a SECO. 
A valuable thing was to collect more information on the SECO platform, as observed in the answer for the third question: "since it does not have a pre-established platform, it is an integration". Such fragment explains why it is harder to determine some dimensions (e.g. environmental) and how the relation between requirements can change over time. Regarding the fourth question, the participant got confused on the SECO name: "if the name 'emergent' goes to this case study, it is correct".

Finally, the opportunity of applying the framework allowed us to use the signs plus (+) and minus (-), as they both were used by the original system sustainability framework (Lago et al. 2015).

\section{Conclusions}

We could observe how sustainability can be analyzed in a real case of emerging SME SECO from the instantiation of the system sustainability framework as well as the results obtained with the study with an expert. We noticed the presence and importance of copying with different sustainability dimensions (notably technical and social, as well as their relations). Moreover, the topic still lacks analysis and modeling tools focused on the software industry. An SME SECO case was studied with the use of that framework to better understand the influence of this requirement in this context. The generated model was evaluated through a semi-structured interview with the purpose of verifying the analysis performed by the researchers. The results show that the technical (and especially social) dimensions of sustainability present a set of factors that may affect the longevity of platforms and/or ecosystems, including the ones who are emerging.

As contributions, we highlight the application of the system sustainability framework in the context of a real SECO case as well as identification of factors that affect the ecosystem longevity, demonstrating the importance of treating 'sustainability' in this scenario. While there is a concern for sustainability, little is known about what is needed to effectively be sustainable in information systems and software engineering. We emphasize that the analysis of a real SECO case contains less information on the environmental dimension if compared to others.

Despite the difficulty in finding data on SME SECO, it was possible to extract information based on an interview with an expert. Another observation is that there is still a lack of understanding how to treat sustainability as a requirement of a platform and/or SECO in general. As future work, we intend to develop a tool to model and analyze a SECO case in order to explore elements of the 'sustainability' requirement. In addition, we intended to carry out case studies with real SECO in order to evaluate the effectiveness and efficiency of this tool in relation to the understanding of the impacts and influences generated by each dimension of sustainability.

\section{Acknowledgments}

This work was supported by UNIRIO.

\section{References}

Barbosa, O., Santos, R., Alves, C., Werner C., and Jansen, S. (2013). "A Systematic Mapping Study on Software Ecosystems from a Three-Dimensional Perspective", In: Jansen, S.; Brinkkemper, S.; Cusumano, M. A. (Org.). Software Ecosystems: 
Analyzing and Managing Business Networks in the Software Industry. 1ed. Cheltenham UK, Northampton USA: Edward Elgar Publishing, 1, 59-81.

Basili, R. V. (1992). "Software modeling and measurement: The Goal/Question/Metric paradigm", Technical Report: Software modeling and measurement: the Goal/Question/Metric paradigm. University of Maryland at College Park College Park, MD, USA.

Bosch, J., and Bosch-Sijtsema P. (2010). "From integration to composition: On the impact of software product lines, global development and ecosystems", The Journal of Systems and Software 83, 1, 67-76.

Capilla, R., Nakagawa, Y. E., Zdun, U., and Carrillo, C. (2017). "Toward Architecture Knowledge Sustainability: Extending System Longevity." IEEE Software 34, 2, 108-111. DOI: 10.1109/MS.2017.54

Fernandes, J., and Santos, R. (2017). "Exploratory Study on Interoperability in the SIGA Ecosystem: An Analysis of the Dimensions of ePING", In: Proceedings of the VIII Workshop on Aspects of Human-Computer Interaction in the Social Web (WAHCISW'17), Joinville, Brazil, 80-81.

Hilty, M. L., Arnfalk, P., Erdmann, L., Goodmand, J., Lehmann, M., and Wägera, A. P. (2006). "The relevance of information and communication technologies for environmental sustainability: A prospective simulation study", Environmental Modelling \& Software 21, 11, 1618-1629.

Jansen, S., Finkelstein, A., and Brinkkemper, S. (2009). "A Sense of Community: A Research Agenda for Software Ecosystems", In: Proceedings of the 31st International Conference on Software Engineering (ICSE'09). New and Emerging Research Track, Vancouver, Canada, 187-190.

Lago, P., Koçak, A. S., Crnkovic, I., and Penzenstadler, B. (2015). "Framing Sustainability as a Property of Software Quality", Communications of the ACM 58, $10,70-78$.

Lago, P., and Penzenstadler, B. (2017). "Editorial: Reality check for software engineering for sustainability - pragmatism required", Journal of Software: Evolution and Process 29, 2, 1-4.

Santos, R. (2016). "Managing and Monitoring Software Ecosystem do Support Demand and Solution Analysis". PhD Thesis, COPPE/UFRJ, Rio de Janeiro, Brazil.

Santos, R., Viana, D., and Maciel, C. (2016). "Ecossistemas de Software: Uma Visão sobre Fatores Técnicos, Humanos e Organizacionais". In: I. Gasparini, M. Mota (Org.) Livro dos Tutoriais do XV IHC, v. C, 70-90. In Portuguese.

Valença, G., Alves, C., Heimann, V., Jansen, S., and Brinkkemper, S. (2014). "Competition and Collaboration in Requirements Engineering: A Case Study of an Emerging Software Ecosystem", In: Proceedings of the IEEE $22^{\text {nd }}$ International Requirements Engineering Conference (RE'14), Karlskrona, Sweden, 384-393. 